\title{
Malignant Cardiac Hemangiopericytoma
}

National Cancer Institute

\section{Source}

National Cancer Institute. Malignant Cardiac Hemangiopericytoma. NCI Thesaurus. Code C5365.

A malignant hemang iopericytoma arising in the heart. 